\title{
Perioperative Anaphylaxis: A different presentation
}

Regufe R., Duarte J. S., Ferreira E., Carvalho R., Azevedo J.

Hospital de São Bernardo, Dept of Anaesthesiology, Setúbal.

\section{Background}

Anaphylaxis is a rare but devastating occurrence during the perioperative period. Its incidence is estimated to be 1 in 3500 to 1 in 20000 surgeries, with a mortality rate currently in the range of $0-1.4 \%$ and an unknown morbidity rate [1;3]. Considering that in induction and maintenance of anaesthesia, multiple drugs are administered in a sequential manner and in short periods of time, there is a higher risk of coming across these severe imune-mediated reactions.

\section{Case Report}

\section{Pre-Operative}

\section{శా, 69 years-old, 58kg, ASA 2}

Right laparoscopic hemicolectomy

Smoking habits and past alcoholism

No previous surgeries

Normal physical examination, blood analysis, ECG and X-ray.

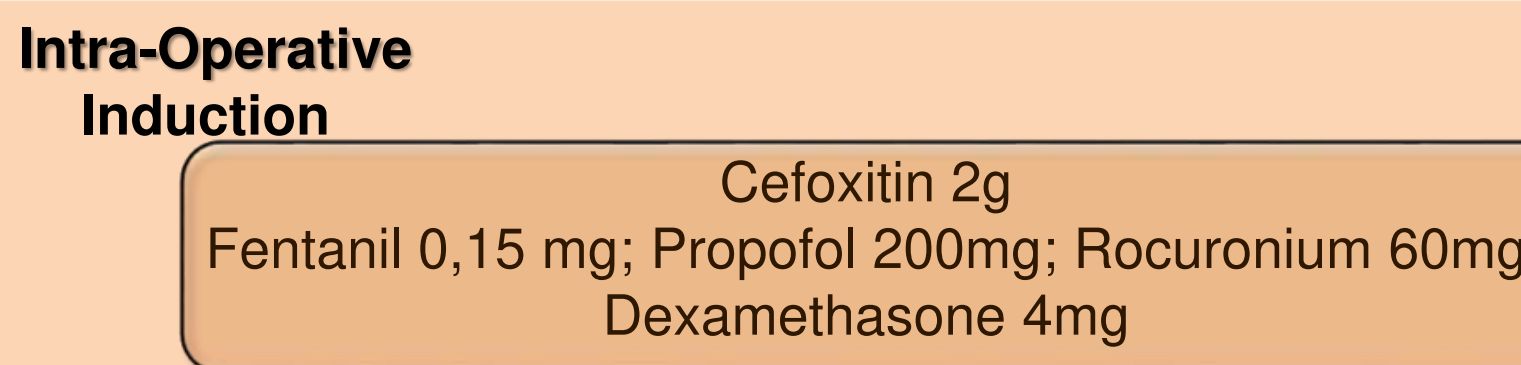

Pallor, sinus tachycardia $\sim 155 \mathrm{bpm}$, end tidal $\mathrm{CO}_{2}$ of $8 \mathrm{mmHg}$ and profound hypotension $\sim 26 / 19 \mathrm{mmHg}$, with normal pulmonary auscultation.

\section{Ephedrine (Total of $30 \mathrm{mg}$ )}

$25 \mathrm{~min} \quad$ Volume expansion with crystalloids

Evaluation by the cardiologist Invasive BP monitoring (radial artery)

\section{Exuberant cutaneous macular rash - face and torso BP 90/50 mmHg, HR $115 \mathrm{bpm}$ Periocular and glottis oedema}

\section{Hydrocortisone $200 \mathrm{mg}$}

Clemastine $4 \mathrm{mg}$

Surgery Postponed

Transfer to ICU intubated

\section{Post-Operative}

Favourable evolution

Serum triptase measurements Anaphylactic reaction

\section{One Month Later}

Right hemicolectomy under general anaesthesia - hydrocortisone, midazolam, fentanyl, etomidate and atracurium. No complications in the perioperative period

\section{Discussion}

Anaphylaxis in the perioperative period:

- Main responsible agents

$\begin{array}{ll}\text { NMBAs } & \begin{array}{l}\text { Rocuronium } \\ \text { Succinylcoline }\end{array} \\ \text { Antibiotics } \\ \text { Latex }\end{array}$

- Cross reactivity is common within NMBAs.

- Allergy may occur upon first exposure.[1;3]

- Can have a diferent presentation from anaphylaxis outside the operating room.

- It's usually not the first diagnostic hypothesis for the anaesthesiologist. [2]

Unless there is a high suspicion of anaphylaxis, the variety of unspecific symptoms and, sometimes, the absence of typical signs that may be masked by the anaesthesia itself or covered in drapes, often lead to a delay in the diagnosis and thus, a delay in the administration of one of the cornerstones of the early treatment: epinephrine.

\section{Ring and Messmer Clinical Scale}

\begin{tabular}{|ll|}
\hline $\begin{array}{l}\text { Grade I } \\
\text { Grade II }\end{array}$ & $\begin{array}{l}\text { Cutaneous-mucous signs } \\
\text { (tachycardia, hypotension); Respiratory signs }\end{array}$ \\
\hline Grade III & Cardiovascular collapse; Bronchospasm \\
\hline Grade IV & Cardiac arrest \\
\hline
\end{tabular}

This clinical pattern is less frequently described amongst Grade III reactions, with cardiovascular collapse associated with tachycardia and absent cutaneous vasodilation during the early stage of anaphylaxis, before hemodynamic restoration. These changes can be due to the activation of the sympathetic nervous system and by the time there is a restoration of peripheral perfusion and cutaneous vasodilation, epinephrine administration should be carefully re-evaluated.[3]

Serum tryptase levels and skin testing are imperative tools in the etiological diagnosis and determining pharmacologic alternatives.

No premedication is proven to prevent further events.

\section{Conclusion}

- The anaesthesiologist should be alert for anaphylaxis in patients with cardiovascular collapse without apparent cause, that doesn't respond properly to the conventional treatment.

- Delays in diagnosis determine delays in the institution of the mainstay treatment - epinephrine and fluid therapy - which can contribute to increase morbidity and mortality.

- Adequate follow up is crucial in investigating the cause and seeking safer alternatives for further anesthetics.

[1].Kannan J. Perioperative Anaphylaxis. Immunol Allergy Clin N Am 2015; 35: 321-334.

[2]. Hepner D. Anaphylaxis during the perioperative period. Anesth Analg 2003; 97: 1381-95.

[3].Dewachter P. Perioperative Anaphylaxis: What should be known?. Curr Allergy Asthma Rep 2015; 15: 21.

Copyright @ 2016 Regufe R., Duarte J.S., Ferreira E., Carvalho R, Azevedo J. rfregufe@ hotmail.com 